\title{
Gold Catalysis in Organic Synthesis: \\ Efficient Cycloisomerization of $\alpha$-Aminoallenes to 3-Pyrrolines
}

\author{
Nobuyoshi Morita and Norbert Krause* \\ Dortmund University, Organic Chemistry II, D-44221 Dortmund, Germany \\ norbert.krause@.uni-dortmund.de
}

\section{Supporting Information}

\section{General Information}

NMR spectra were recorded with a Bruker WM 400 spectrometer at $400 \mathrm{MHz}\left({ }^{1} \mathrm{H}\right)$ and 100.6 $\mathrm{MHz}\left({ }^{13} \mathrm{C}\right)$ in $\mathrm{CDCl}_{3}$ as solvent. The signals of the major component of a product mixture are marked with an asterisk (*). GC analyses were carried out with a Carlo Erba GC 8000 gas chromatograph with helium as the carrier gas and an OV-1701 capillary column.

\section{Typical procedure}

$20 \mu \mathrm{L}$ (2 mol\%) of a solution of $\mathrm{AuCl}_{3}$ in $\mathrm{CH}_{3} \mathrm{CN}(0.165 \mathrm{M})$ was added to a solution of the $\alpha$-aminoallene $4 a$ (42 $\mathrm{mg}, 0.17 \mathrm{mmol}$ ) in $5 \mathrm{ml}$ of dry $\mathrm{CH}_{2} \mathrm{Cl}_{2}$ at room temperature under argon. After 5 days (the reaction was monitored by thin layer chlomatography), the solvent was removed in vacuo and the crude product submitted to $\mathrm{SiO}_{2}$ column chromatography ( $\left.\mathrm{MeOH} / \mathrm{CH}_{2} \mathrm{Cl}_{2}, 1 / 10\right)$ to give 3-pyrroline $5 a$ (31 mg, 74\%).

\section{2-Benzyloxymethyl-5-isopropyl-3-methyl-2,5-dihydro-1H-pyrrole (5a)}

${ }^{1} \mathrm{H}-\mathrm{NMR} \delta$ 7.35-7.27 (m, 5H), 5.46 (d, J = 1.5 Hz, 1H), 4.57 (ABq, J = $\left.12.0 \mathrm{~Hz}, 2 \mathrm{H}\right)$, 4.10-4.09 (m, 1H), 3.88-3.83 (m, 1H), 3.63 (dd, J = 9.8, 3.5 Hz, 1H), 3.45 (dd, J = 9.8, $5.8 \mathrm{~Hz}, 1 \mathrm{H}$ ), 1.99 (br s, 1H), 1.70 (s, 3H), 0.94 (d, J = $6.8 \mathrm{~Hz}, 3 \mathrm{H}$ ), 0.90 (d, J = 6.8 Hz, 3H). ${ }^{13} \mathrm{C}-\mathrm{NMR} \delta$ 138.3, 137.6, 128.5, 127.9, 127.7, 125.7, 73.3, 71.2, 70.6, 67.3, 33.7, 19.4, 19.0, 13.7 .

\section{2-Benzyloxymethyl-5-isopropyl-1-methanesulfonyl-3-methyl-2,5-dihydro-1H-} pyrrole (5b)

${ }^{1} \mathrm{H}-\mathrm{NMR} \delta$ 7.37-7.27 (m, 5H), 5.43 (d, J = $\left.1.5 \mathrm{~Hz}, 1 \mathrm{H}\right), 4.53(\mathrm{ABq}, \mathrm{J}=12.0 \mathrm{~Hz}, 2 \mathrm{H})$, 4.41-4.39 (m, 1H), 4.38-4.34 (m, 1H), 4.24 (dd, J = 11.0, $2.2 \mathrm{~Hz}, 1 \mathrm{H}), 3.52$ (dd, J = 11.0, $2.0 \mathrm{~Hz}, 1 \mathrm{H}), 2.92$ (s, 3H), 2.63-2.55 (m, 1H), 1.67 (d, J = 0.8 Hz, 3H), 0.90 (d, J = 
$7.3 \mathrm{~Hz}, 3 \mathrm{H}), 0.78$ (d, J = 6.8 Hz, 3H). ${ }^{13} \mathrm{C}-\mathrm{NMR} \delta$ 137.6, 136.3, 128.6, 128.0, 127.9, 121.1, 73.2, 71.9, 70.8, 66.3, 39.3, 31.1, 19.5, 15.1, 13.6 .

\section{2-Benzyloxymethyl-5-isopropyl-3-methyl-1-(toluene-4-sulfonyl)-2,5-dihydro-1H- pyrrole (5c)}

${ }^{1} \mathrm{H}-\mathrm{NMR} \delta 7.75$ (d, J = 8.3 Hz, 2H), 7.35-7.10 (m, 7H), 5.37 (d, J = 1.5 Hz, 1H), 4.524.49 (m, 1H), 4.48-4.44 (m, 1H), 4.17 (ABq, J = 12.0 Hz, 2H), 4.01 (dd, J = 10.6, 2.8 Hz, 1H), 3.55 (dd, J = 10.6, 2.0 Hz, 1H), 2.73-2.67 (m, 1H), 2.33 (s, 3H), 1.66 (s, 3H), 0.90 (d, J = 7.0 Hz, 3H), 0.64 (d, J = $6.5 \mathrm{~Hz}, 3 \mathrm{H}) .{ }^{13} \mathrm{C}-\mathrm{NMR} \delta 142.6,139.4,138.2$, 136.5, 129.3, 128.3, 127.6, 127.5, 126.8, 120.9, 72.8, 72.6, 70.4, 67.4, 30.7, 21.5, 19.6, 15.2, 13.8 .

\section{1-(2-Benzyloxymethyl-5-isopropyl-3-methyl-2,5-dihydro-pyrrol-1-yl)-ethanone} (5d)

${ }^{1}$ H-NMR $\delta$ 7.34-7.22 (m, 5H), 5.43 / 5.38* (d, J = 1.2 Hz, 1H), 4.68-4.63 / 4.55-4.50* (m, 1H), 4.49* (ABq, J = $12.3 \mathrm{~Hz}, 2 \mathrm{H}), 4.49$ (s, 2H),4.49-4.45* / 4.40-4.35 (m, 1H), 4.24 / 3.59* (dd, J = 10.0, 3.0 Hz, 2H), 2.90-2.82 / 2.22-2.15* (m, 1H), 2.06* / 2.00 (s, 3H), 1.78 / 1.74* (s, 3H), 0.93* / 0.89 (d, J = 7.0 Hz, 3H), 0.67* / 0.56 (d, J = 6.8 Hz, $3 \mathrm{H}) .{ }^{13} \mathrm{C}-\mathrm{NMR} \delta 169.7^{*}, 169.3,138.7^{*}, 137.9^{*}, 137.8,136.4,128.6^{*}, 128.4^{*}, 128.0$, 127.8, 127.6*, 127.6, 121.7, 119.8*, 73.4, 73.2*, 70.9, 70.1*, 70.0, 68.6, 68.1*, 65.8*, $32.0^{*}, 26.5,22.9 *, 19.5,19.4^{*}, 15.0,14.8^{*}, 14.2,13.9 *$.

\section{tert-Butyl 2-benzyloxymethyl-5-isopropyl-3-methyl-2,5-dihydro-pyrrole-1-} carboxylate (5e)

${ }^{1} \mathrm{H}-\mathrm{NMR} \delta$ 7.35-7.24 (m, 10H), 5.40 (d, J = 1.2 Hz, 1H), 5.36 (d, J = 1.3 Hz, 1H), 4.55 (ABq, J = 12.3 Hz, 2H), 4.50 (s, 2H), 4.49-4.46 (m, 1H), 4.40-4.35 (m, 1H), 4.34-4.31 (m, 1H), 4.28-4.25 (m, 1H), 4.16 (dd, J = 10.0, 3,2 Hz, 1H), 3.83 (dd, J = 10.0, $4.0 \mathrm{~Hz}$, $1 \mathrm{H})$, 3.65-3.59 (m, 2H), 2.82-2.74 (m, 1H), 2.53-2.45 (m, 1H), 1.75 (s, 3H), 1.74(s, 3H), 1.50 (s, 9H), 1.41 (s, 9H), 0.89 (d, J = 7.0 Hz, 3H), 0.88 (d, J = 7.0 Hz, 3H), 0.64 (d, J = $6.8 \mathrm{~Hz}, 3 \mathrm{H}), 0.62$ (d, J = 6.8 Hz, 3H). ${ }^{13} \mathrm{C}-\mathrm{NMR} \delta$ 153.9, 153.3, 138.9, 138.5, 137.2, 137.1, 128.5, 128.4, 127.7, 127.6, 127.5, 121.1, 120.8, 79.3, 79.2, 73.5, 73.3, 69.6, 69.3, 68.9, 67.9, 67.6, 66.6, 29.8, 28.7, 27.5, 27.0, 19.5, 19.3, 15.1, 15.0, 14.2, 14.0,

\section{2-Benzyloxymethyl-3,5-dimethyl-2,5-dihydro-1H-pyrrole (7a)}

${ }^{1} \mathrm{H}-\mathrm{NMR} \delta$ 7.36-7.27 (m, 5H), 5.40 (br s, 1H), 4.59 (ABq, J = 11.8 Hz, 2H), 4.40-4.32 (m, 1H), 4.30-4.26 (m, 1H), 3.71 (dd, J = 10.0, 3.3 Hz, 1H), 3.54 (dd, J = 10.0, $5.3 \mathrm{~Hz}$, 
1H), 1.69 (s, 3H), $1.36(\mathrm{~d}, \mathrm{~J}=6.6 \mathrm{~Hz}, 3 \mathrm{H}) .{ }^{13} \mathrm{C}-\mathrm{NMR} \delta$ 138.0, 135.8, 128.5, 128.0, 127.8, 127.7, 73.4, 69.1, 66.9, 60.4, 20.5, 13.4 .

2-(tert-Butyl-dimethyl-silanyloxymethyl)-5-hexyl-3-methyl-2,5-dihydro-1H-pyrrole (7b)

${ }^{1} \mathrm{H}-\mathrm{NMR} \delta 5.43$ (br s, 1H), 4.08-4.00 (m, 2H), 3.82 (dd, J = 10.6, 3.7 Hz, 1H), 3.65 (dd, $\mathrm{J}=10.6,4.3 \mathrm{~Hz}, 1 \mathrm{H}), 1.71$ (s, 3H), 1.62-1.52 (m, 2H), 1.39-1.25 (m, 8H), 0.90-0.85 (m, 3H), 0.87 (s, 9H), 0.07, 0.05 (2 s, 6H). ${ }^{13} \mathrm{C}-\mathrm{NMR} \delta$ 137.1, 126.9, 68.6, 64.6, 63.4, 31.9, 29.4, 26.6, 26.0, 22.8, 18.4, 14.2, 13.7, -5.2, -5.4.

2-(tert-Butyl-dimethyl-silanyloxymethyl)-3-methyl-5-phenyl-2,5-dihydro-1Hpyrrole (7c)

${ }^{1}$ H-NMR $\delta$ 7.30-7.22 (m, 5H), 5.50 (br s, 1H), 5.04 (br s, 1H), 4.05 (br s, 1H), 3.74 (dd, $\mathrm{J}=$ 8.0, $3.4 \mathrm{~Hz}, 1 \mathrm{H}$ ), 3.62 (dd, J = 8.0, $4.6 \mathrm{~Hz}, 1 \mathrm{H}), 1.81$ (s, 3H), 0.91 (s, 9H), 0.09 (s, 6H). ${ }^{13} \mathrm{C}-\mathrm{NMR} \delta 145.2,139.1,128.6,127.8,127.2,127.0,69.5,67.8,65.9,26.0,18.4$, 13.9, $-5.2,-5.3$. 